\title{
Caracterización de una zona de contacto parapátrico entre Astrocaryum macrocalyx y Astrocaryum urostachys en el límite entre la planicie Marañón-Pastaza y el Arco de lquitos
}

\section{Characterization of a parapatric contact zone between Astrocaryum macrocalyx and Astrocaryum urostachys at the boundary of the Marañón-Pastaza flood plain and the lquitos arch}

\section{Víctor Vargas Paredes ${ }^{1}$ y Jean-Christophe Pintaud ${ }^{2}$}

1 Institut de Recherche pour le Développement (IRD), Perú. Apartado postal: Casilla 18-1209, Lima 18, Perú.

2 Institut de Recherche pour le Développement (IRD), Perú. UMR DiA-PCIDYNADIV, BP 64501 DIA-PCIDYNADIV, BP 64501 34394 Montpellier Cedex 5, France.

Email Jean-Christophe Pintaud: jean-christophe.pintaud@ird.fr
Trabajo presentado al Simposio Internacional "LAS PALMERAS EN EL MARCO DE LA INVESTIGACIÓN PARA EL MARCO DE LA INVESTIGACION PARA EL" del 07 a 09 de Novi Museo de Historia Natural, UniMuseo de Historia Natura, Universidad Nacional Mayor de San Marcos, Lima, Perú.

Publicado online: 29/11/2008

\section{Resumen}

Se determinó una zona de contacto parapátrico entre Astrocaryum macrocalyx y Astrocaryum urostachys en el límite entre la planicie Marañón-Pastaza y el Arco de lquitos, en la Amazonía norperuana. Para ello se realizó un muestreo de 35,5 hectáreas. Esta área de estudio se dividió en 9 transectos con dimensiones de 1 a $3 \mathrm{~km}$ por $20 \mathrm{~m}$ de ancho. Se tomaron datos de abundancia, altura de la planta y variaciones topográficas a lo largo de los transectos. Los resultados indican que estas especies entran en contacto solamente en una pequeña área en la zona de confluencia de los Ríos Marañón y Tigre en la margen suroccidental del arco de lquitos. Durante la evaluación se registró un total de 1133 individuos del género Astrocaryum. La mayoría de individuos $(58,5 \%)$ se ubican en las clases de tamaño inferiores $(0-3,90 \mathrm{~m}$ de alto) mientras que los individuos mayores a $12 \mathrm{~m}$ representan $9,2 \%$ de los individuos censados. En las tres posiciones topográficas (pendientes, valles y colinas) la mayor cantidad de individuos están presentes en las laderas y en los zonas bajas de las colinas.

Palabras Clave: Astrocaryum urostachys, Astrocaryum macrocalyx, arco de lquitos, contacto parapátrico, transectos.

\section{Abstract}

This study identifies a parapatric contact zone between Astrocaryum macrocalyx and Astrocaryum urostachys at the boundary between the Marañón-Pastaza flood plain and the Iquitos Arch, in the northern Peruvian Amazon. The area sampled covers 35,5 ha in the form of 9 transects $1-3 \mathrm{~km}$ long $\times 20 \mathrm{~m}$ wide. All Astrocaryum individuals were recorded within the transects, and their size was measured as well as topographical data of the environment. The results show that the two species meet only in a small area in the confluence zone of Marañón and Tigre rivers near the southwest basement of the lquitos Arch. A total of 1133 Astrocaryum individuals were recorded, mostly in the $0-3,90 \mathrm{~m}$ size classes, with only $9,2 \%$ of the individuals exceeding $12 \mathrm{~m}$ in high. Within the three topographical positions considered (slope, valley bottom and ridge), most individuals were found in the lower slopes and bottoms of valleys.

Keywords: Astrocaryum urostachys, Astrocaryum macrocalyx, Iquitos arch, parapatric contact, transects.

\section{Introducción}

Astrocaryum es un género de palmeras neotropicales distribuido particularmente en los bosques y sabanas de la cuenca del Amazonas, extendiéndose hacia el noroeste por los valles interandinos hasta la vertiente pacífica de Colombia y Ecuador, continuando por Centroamérica hasta Costa Rica; hacia el sur se extiende por la periferia sur de la cuenca amazónica hasta los cerrados de Bolivia y Brasil y el bosque atlántico brasileño (Henderson et al. 1995).

El género Astrocaryum se divide en tres subgéneros: Atergynanthus, Pleiogynanthus y Monogynanthus. Las dos especies consideradas Astrocaryum macrocalyx y $A$. urostachys son estrechamente emparentadas (Pintaud \& Kahn 2002). Ambas pertenecen al subgénero Monogynanthus, sección Huicungo, subsección Sachacungo (Kahn, 2008). La sección Huicungo es muy diversificada en el oeste de la cuenca amazónica, con 14 especies, de las cuales 10 se encuentran en el Perú.

Astrocaryum urostachys Burret es una palmera de sotobosque o subdosel que alcanza los $10 \mathrm{~m}$ de alto, multicaula, monoica y con espinas en los frutos, las hojas y el estípite. Se encuentra distribuida en la Amazonía occidental y en el pie de monte andino, en bosques de tierra firme y depósitos aluviales ribereños, en la mayor parte del Oriente Ecuatoriano (Borchsenius et al. 1998; Kahn \& Millán 1992), en las regiones adyacentes de Perú (Amazonas, Loreto) al norte del río Marańón, y en Colombia cerca del río Putumayo (Kahn \& Gluchy 2002), entre 100 y 1000 m de altura.

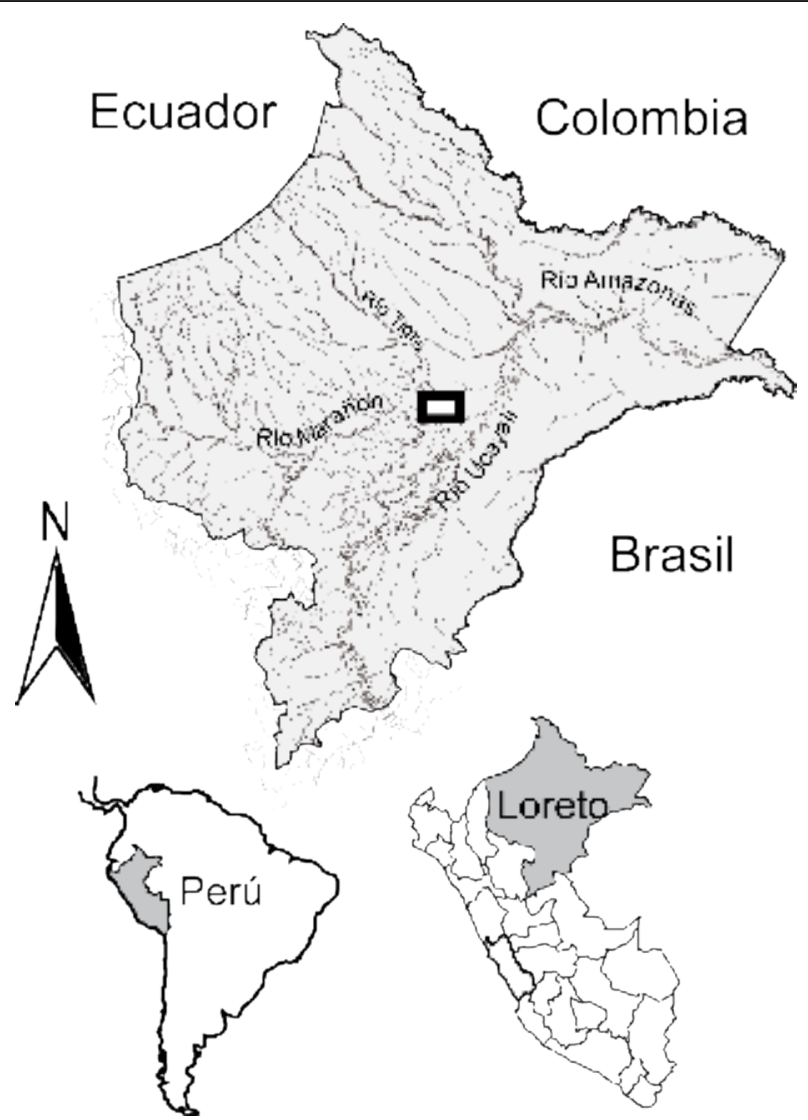

Figura 1. Mapa de la Región Loreto y ubicación de la zona de estudio 


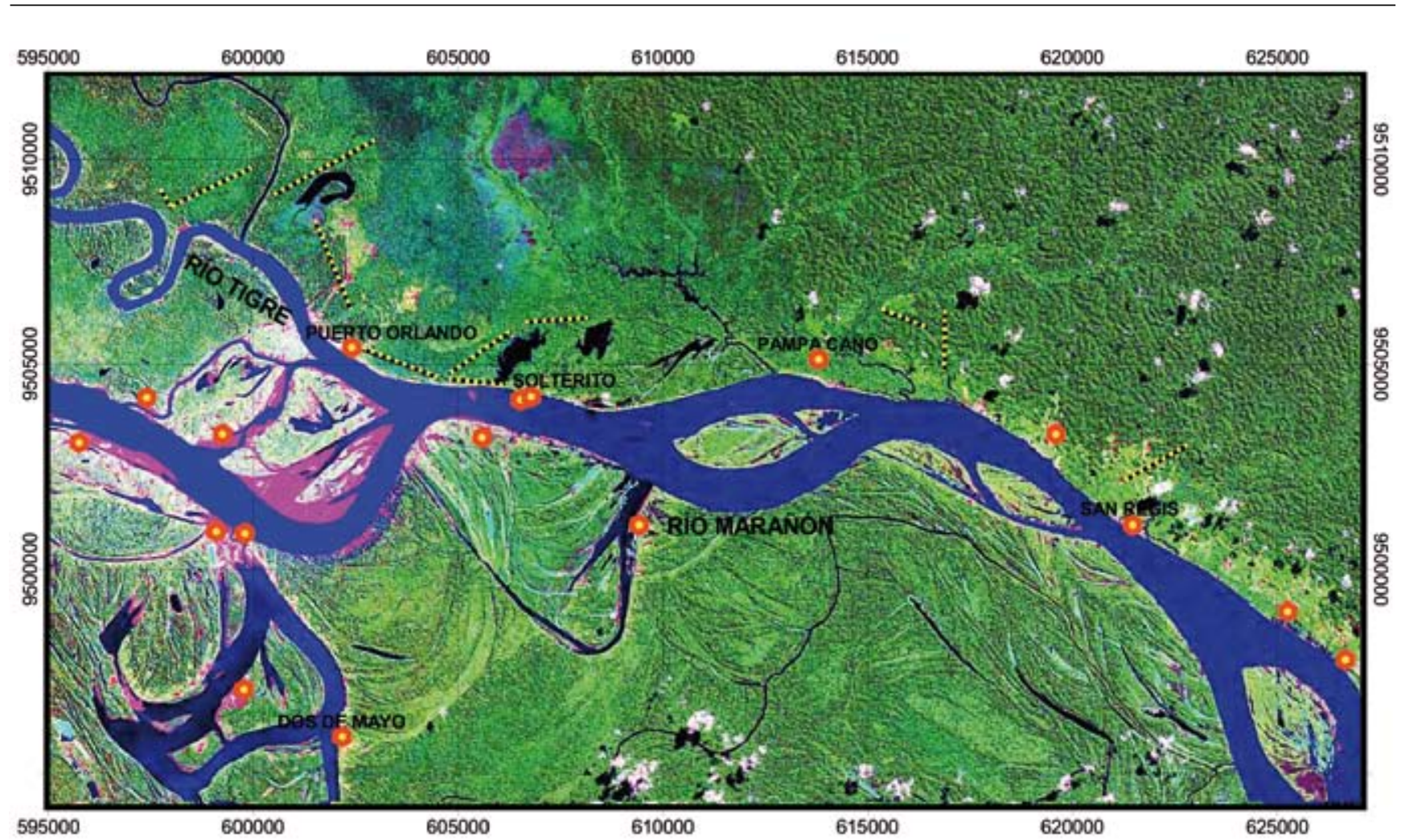

Figura 2. Imagen de satélite Landsat ubicando el lugar de muestreo y los transectos realizados (líneas amarillas punteadas)

Astrocaryum macrocalyx Burret es una palma de tamaño medio con un estípite que llega a los $10 \mathrm{~m}$, monocaula, monoica y con espinas en frutos, hojas y estípite. Está distribuida en los bosques de altura (tierra firme) de la parte norte de la Amazonía peruana (Kahn \& Moussa 1994; Pintaud et al. 2003).

El efecto de las barreras geográficas, y en particular los ríos y arcos, sobre la dinámica de la biodiversidad en la Amazonía occidental ha sido resaltado por varios autores (Räsänen $e t$ al. 1987, Hooghiemstra 2002). En este estudio presentamos un ejemplo de relación entre estas estructuras y la distribución de dos especies afines de palmeras.

\section{Material y métodos}

El estudio se realizó en la zona de la confluencia del río Tigre con el Marañón en los bosques de las comunidades de Miraflores (río Tigre), Puerto Orlando (confluencia Tigre - Marañón), Solterito, Pampacaño y el Fundo Iquitos, en el Río Marañón, todos en el departamento de Loreto (Fig. 1-2).

El muestreo se realizó en un área de 17,75 km de largo x $20 \mathrm{~m}$ de ancho (35,5 hectáreas), distribuidas en 9 transectos de uno a tres kilometros de largo, divididos en subunidades de 50 × $20 \mathrm{~m}$. En cada subunidad se registró la abundancia de los individuos del género Astrocaryum con sus respectivas alturas. En el caso de Astrocaryum urostachys, que es una especie cespitosa, cada tallo se consideró como un individuo. Adicionalmente, para determinar el perfil topográfico, se tomó medidas de las variaciones topográficas con un clinómetro a lo largo de los transectos en diferentes subunidades dependiendo de la fisiografía del terreno. Para relacionar la topografía con las palmeras se clasificó a la topografía en tres categorías: pendientes, valles y colinas (Vallejo et al. 2004).

\section{Resultados}

Durante la evaluación se registró un total de 1133 individuos del género Astrocaryum, de los cuales 917 corresponden a la especie de Astrocaryum macrocalyx y 216 de Astrocaryum urostachys (Tabla 1).

La mayor cantidad de individuos están entre las alturas de 0-3,90 m (Fig. 3), lo que corresponde al 58,5\% del total de los individuos registrados, mientras que los individuos que alcanzan alturas mayores a $12 \mathrm{~m}$ conforman el 9,2\% de la población.

Astrocaryum urostachys y Astrocaryum macrocalyx se presentan juntos en una zona estrecha en torno a la comunidad de Puerto Orlando. Conforme nos dirigimos río abajo, Astrocaryum uro-

Tabla 1. Número de individuos de Astrocaryum urostachys y Astrocaryum macrocalyx en las comunidades donde se realizó los transectos (Comunidad de Miraflores río Tigre, Pto. Orlando, Solterito, Pampa Caño y Fundo lquitos río Marañón).

\begin{tabular}{lccccccc}
\hline Especie & \multicolumn{5}{c}{ Comunidad } & & \\
\cline { 2 - 7 } & Miraflores & Pto. Orlando & Solterito & Pampa caño & Fundo Iquitos & Total \\
\hline Astrocaryum urostachys & 32 & 159 & 25 & 0 & 0 & 216 \\
Astrocaryum macrocalyx & 0 & 131 & 465 & 108 & 213 & 917 \\
\hline
\end{tabular}




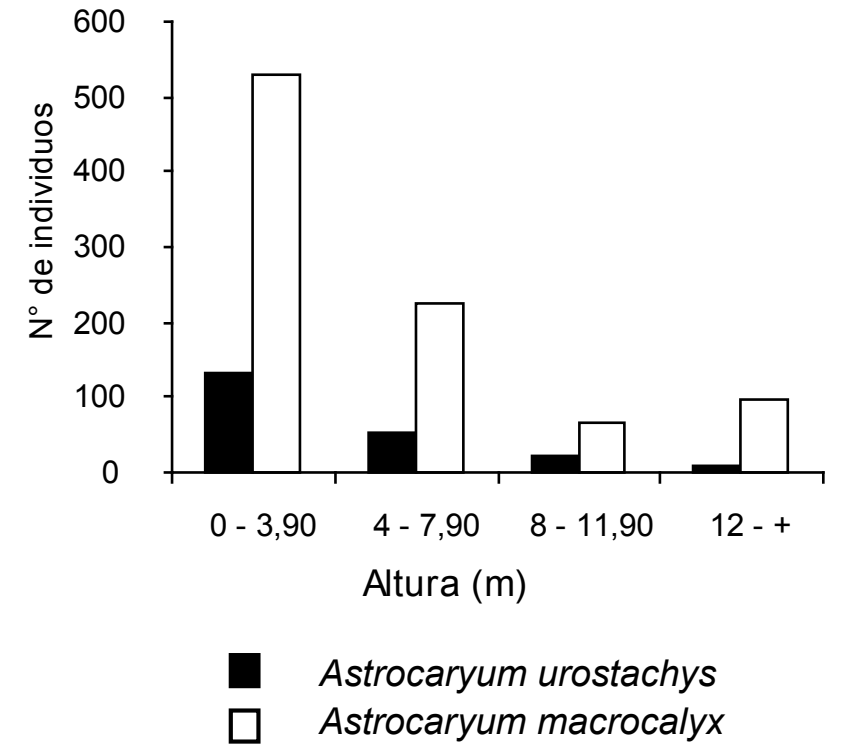

Figura 3. Número de individuos de Astrocaryum urostachys y Astrocaryum macrocalyx con relación a los diferentes rangos de altura.

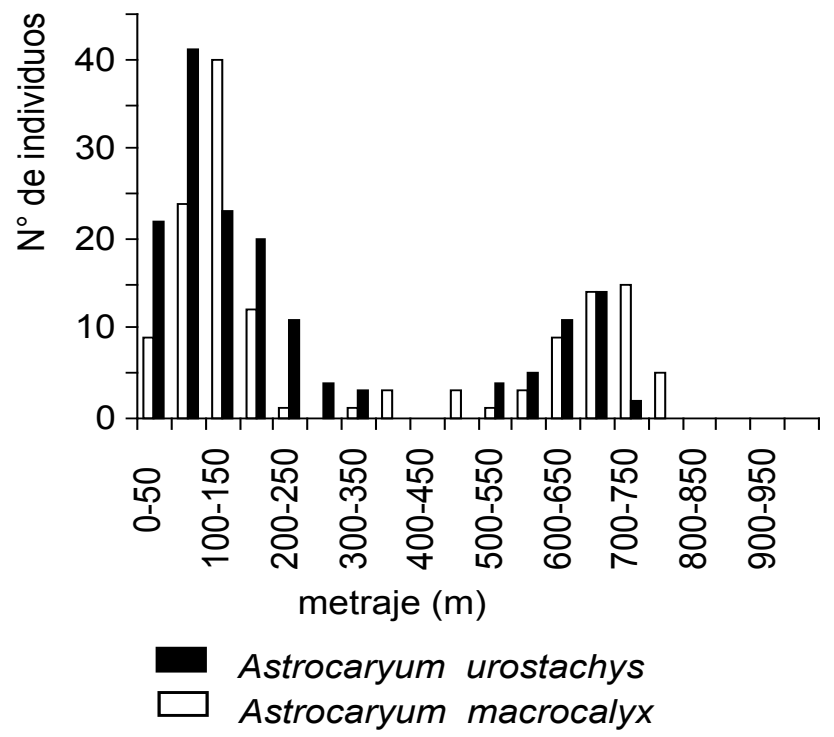

Figura 4. Número de individuos de las dos especies en la franja de simpatría entre Astrocaryum macrocalyx y Astrocaryum urostachys en la zona de la comunidad de Puerto Orlando.

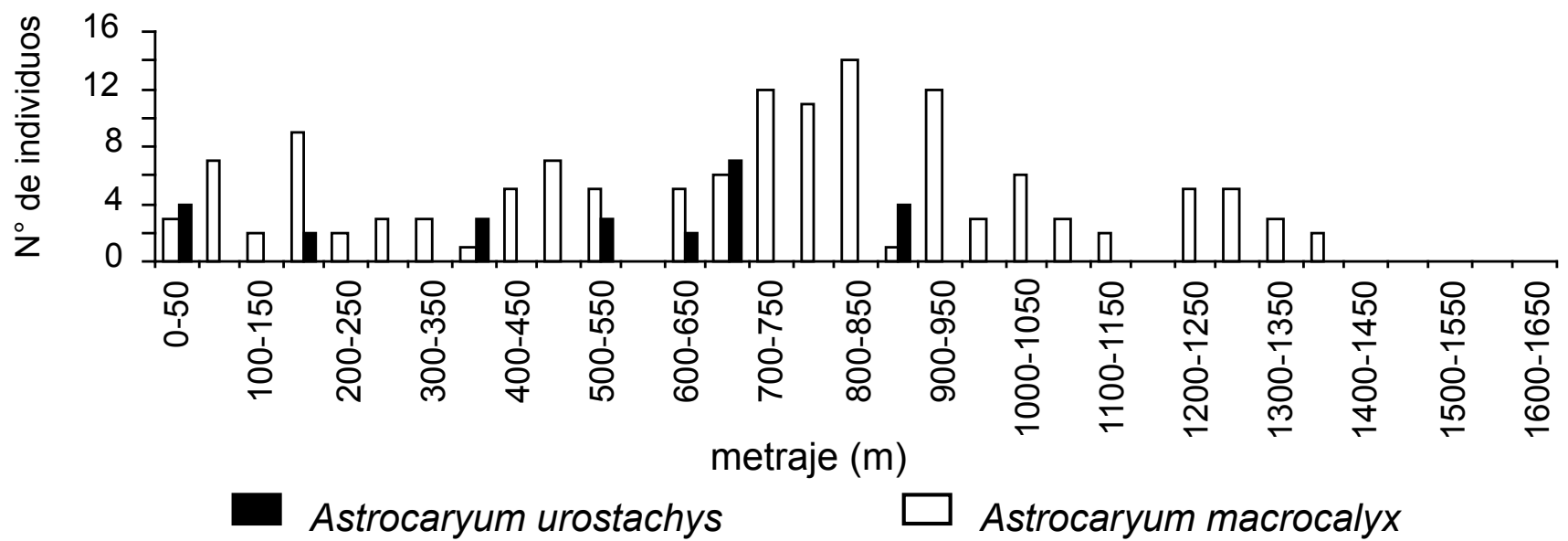

Figura 5. Número de individuos de ambas especies en el límite de la franja de simpatría entre Astrocaryum macrocalyx y Astrocaryum urostachys en la zona de la comunidad de Solterito, donde Astrocaryum urostachys tiende a desaparecer, para sólo registrarse Astrocaryum macrocalyx.

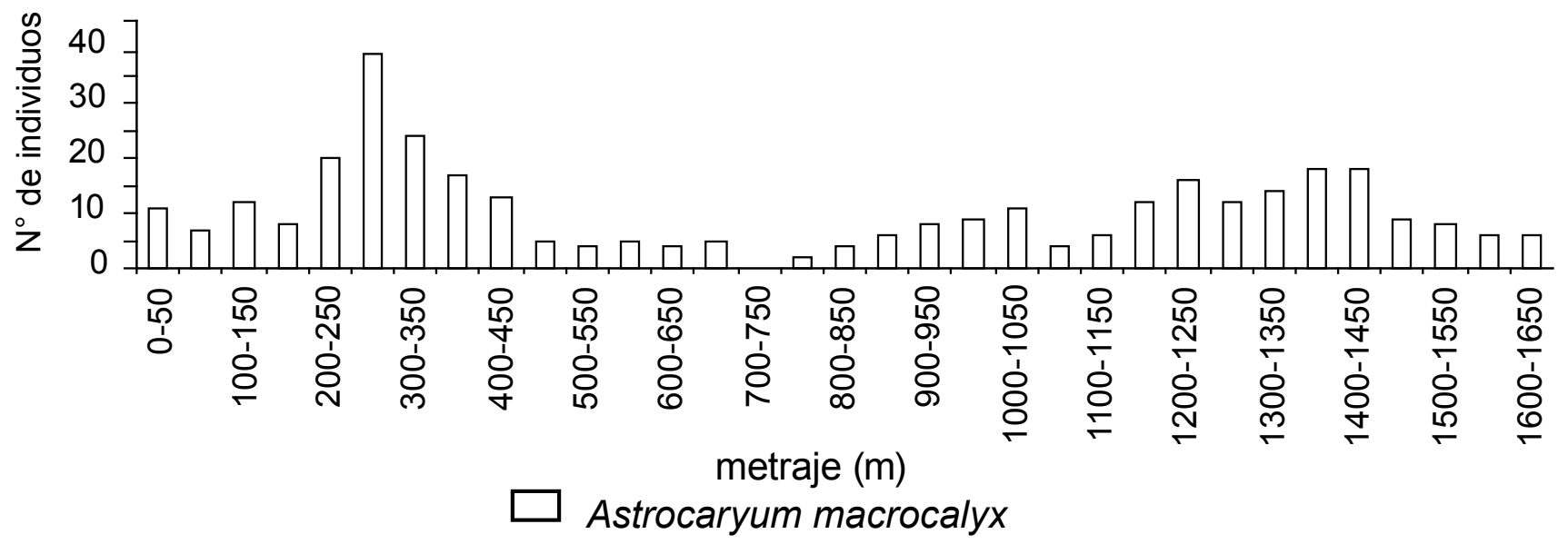

Figura 6. Número de individuos de Astrocaryum macrocalyx en la comunidad de Solterito, donde Astrocaryum urostachys esta ausente y sólo se registró Astrocaryum macrocalyx durante todo el largo del transecto. 


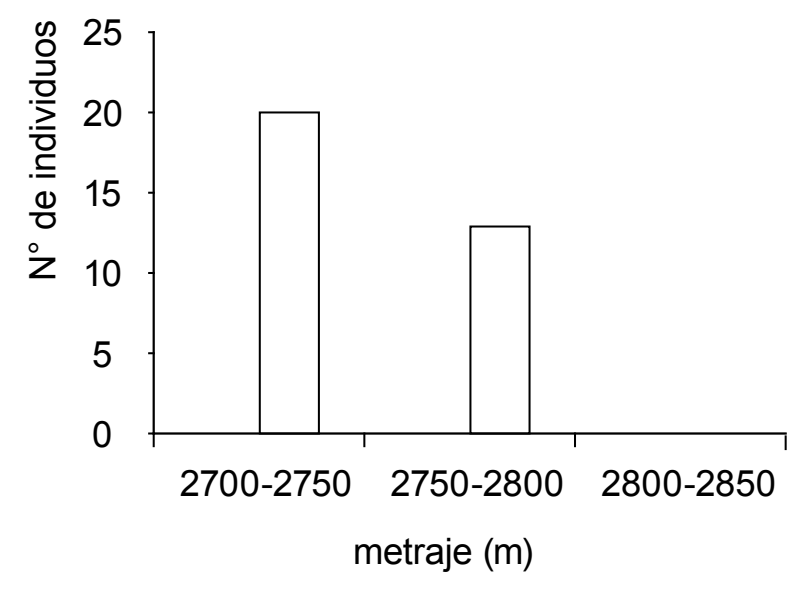

Figura 7. Comunidad de Miraflores, donde se registró Astrocaryum urostachys, mientras que Astrocaryum macrocalyx es ausente.

stachys tiende a desaparecer, para posteriormente registrarse sólo Astrocaryum macrocalyx en todas las zonas posteriores de muestreo. Sin embargo, río arriba del contacto (río Tigre) Astrocaryum macrocalyx desaparece para sólo registrarse Astrocaryum urostachys (Figs. 4-7)

En el transecto realizado en el fundo Iquitos se tomó datos topográficos, los cuales muestran que estas especies se encuentran presentes en las tres posiciones topográficas (pendientes, valles y colinas). El mayor número de individuos se encuentra en las laderas y en las zonas bajas de las colinas, mientras que en las mesetas hay menor abundancia (Fig. 8)

Estas especies se distribuyen de forma dispersa, pudiéndose encontrar un individuo a muchos metros de distancia del más cercano o en forma agrupada, denominándose a esta agrupación como huicungales, cuando en una determinada área se encuentran muchos individuos de la misma especie.

\section{Discusión}

La mayor cantidad de individuos de estas especies se encuentran entre las plántulas y los juveniles que alcanzan el 58,5\% del total de individuos registrados. Muy pocos de éstos llegan a alcanzar el estado adulto, ya sea por el consumo de las semillas por los roedores, por el ataque de algunas especies de insectos, o por la escasa luz que ingresa al sotobosque. También su escasez se puede atribuir al uso que algunos pobladores hacen de las hojas para la construcción del techado de sus viviendas. En estudios similares, (Vallejo et al., 2004), los datos obtenidos del número de individuos de cada estado de desarrollo, hacen suponer que hay una gran mortalidad de plántulas, en contraste con el menor porcentaje de adultos encontrados, posiblemente debido a depredación por parte de herbívoros. En otro estudio hecho por Charles-Dominque et al. (2003) se observó que los individuos juveniles de Astrocaryum sciophilum son depredados por orugas de mariposas. Según Kahn \& Granville (1992) para el crecimiento de las palmeras se requiere diferentes cantidades de luz. Estos autores diferenciaron dos fases de crecimiento: una fase en la que se requiere poca luz para que las plántulas incrementen su tamaño y número de hojas, y otra fase en la que necesita mayor cantidad de luz para que se desarrolle un estípite.

En el transecto donde se tomaron datos topográficos, estas especies habitan en las tres posiciones topográficas, encontrándose la mayor cantidad de individuos en las laderas de las colinas y en los valles, siendo las cimas de las colinas el lugar donde se registró el menor número de individuos. Al parecer estas especies tienen preferencia por aquellos lugares por donde discurre el agua o constantemente se encuentra húmedo, como los valles. Vallejo et al. (2004) mencionan que estas dos especies hermanas tienen la misma preferencia de hábitat, que es justamente el que ocurre en las partes bajas de las colinas, cuyos suelos son hidromórficos, y donde reciben la mayor cantidad de luz. Estos suelos son óptimos para el crecimiento de estas palmeras.

Astrocaryum urostachys y Astrocaryum macrocalyx son especies que desarrollan un comportamiento parapátrico. Sin embargo, se ha caracterizado un contacto con mezcla entre estas dos especies. Este contacto se da en la zona de la confluencia de los ríos Marañón y Tigre, al margen del arco de Iquitos. Las dos especies comparten una pequeña área en la cual la abundancia de una especie no es muy diferente de la otra: Astrocaryum urostachys tiene 159 individuos y Astrocaryum macrocalyx 131 individuos. Estudios anteriores (Pintaud 2005, Montufar \& Pintaud 2006) determinaron el limite de distribución de Astrocaryum urostachys y Astrocaryum macrocalyx en el sector del arco de Iquitos. Las dos especies se encontraron en el borde del arco de Iquitos, Astrocaryum urostachys localizándose a la periferia de esta estructura, mientras que Astrocaryum macrocalyx crece adentro. Dos puntos

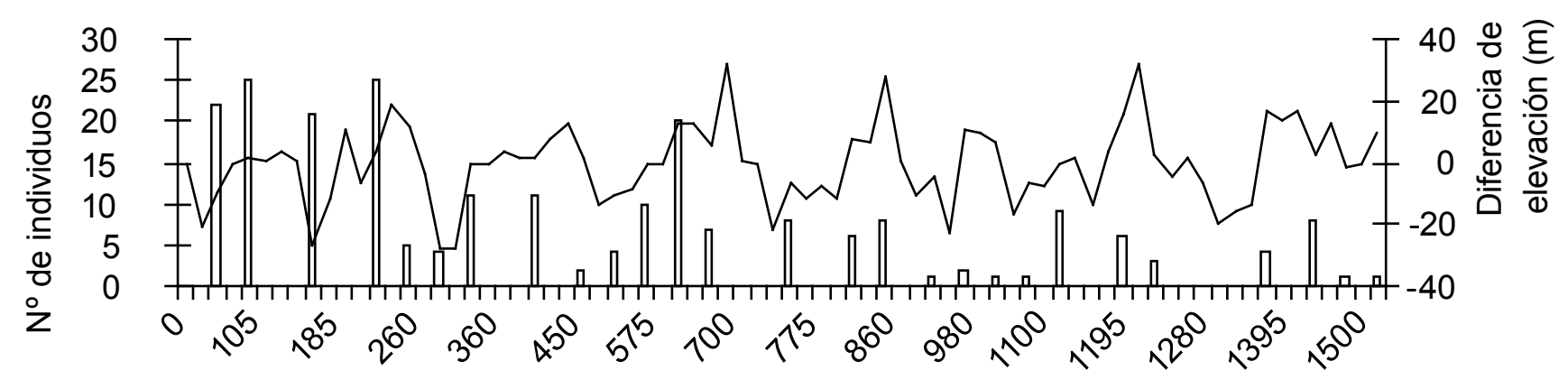

Metraje (m)

Figura 8. Numero de individuos de Astrocaryum macrocalyx en relación a las diferentes posiciones topográficas (pendientes, valles y colinas) tomadas en un bosque de tierra firme. 


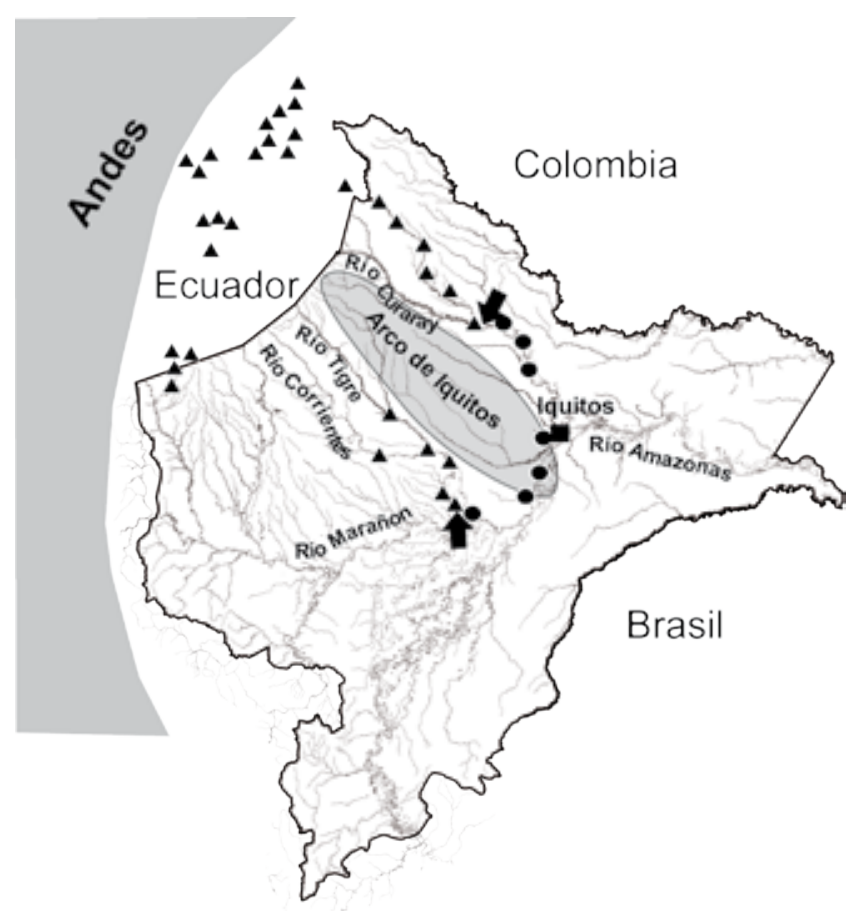

Figura 9. Distribución espacial de Astrocatyum urostachys (triángulos), A. macrocalyx (círculos) en la Amazonía occidental, las flechas indican la zona de contacto o límite entre $A$. urostachys y $A$. macrocalyx, el área gris representa el Arco de lquitos.

de contacto entre las dos especies fueron determinados, el que constituye el objeto del presente estudio y otro localizado cerca de la confluencia entre el río Curaray y el río Napo (Fig. 9). $A$. urostachys ha sido considerado durante mucho tiempo como endémico a Ecuador Oriental y $A$. macrocalyx a los alrededores de Iquitos (Kahn \& Millán, 1992). Sin embargo, estudios posteriores (Kahn \& Gluchy 2002; Montufar \& Pintaud 2006) determinaron que $A$. urostachys tiene un rango de distribución abarcando el noroeste de Loreto, llegando a la margen norte del río Marañón y alcanzando el territorio colombiano a nivel del río Putumayo. La distribución de Astrocaryum macrocalyx, por su parte, se extiende aparentemente en todo el arco de Iquitos al borde del Marañón y posiblemente mas allá en dirección del noreste. En las zonas de contacto entre $A$. urostachys y $A$. macrocalyx las poblaciones de ambas especies forman una estrecha franja de simpatría, al contacto de la planicie de inundación Marañón-Pastaza con el sótano del Arco de Iquitos.

\section{Agradecimientos}

Nuestro agradecimiento al Blgo. Vicente Vásquez, al Bar. Ytalo Mesones por el apoyo en el trabajo de campo y a todos los pobladores de las comunidades por la asistencia en el campo.

\section{Literatura citada}

Borchsenius F., H. Borgtoft Pedersen \& H. Balslev. 1998. Manual to the Palms of Ecuador. Aarhus, University Press. 227 pp.

Charles-Dominique, P., J. Chave, M.A. Dubois, J.J. de Granville, B. Riera \& C.Vezzoli. 2003. Colonization front of the understory palm Astrocaryum sciophilum in a pristine rain forest of French Guiana. Global Ecology and Biogeography 12: 237-248.

Henderson A. 1995. The palms of the Amazon. Oxford University Press.

Henderson A., G. Galeano \& R. Bernal. 1995. Field Guide to the Palms of the Americas. Princenton University Press. New Jersey, U.S.A.

Hooghiemstra H. 2002. The dynamic rainforest ecosystem on geological, quaternary and human time scales. In Verweij P. (ed.), Understanding and capturing the multiple values of tropical forests. Proceedings of the international seminar on valuation and innovative financing mechanisms in support of conservation and sustainable management of tropical forests, pp 7-19.

Kahn F. 2008. The genus Astrocaryum (Arecaceae). Rev peru biol. 15(supl. 1): 031-048.

Kahn F. \& D. Gluchy. 2002. Variation in morphology of the pistillate flowers of Astrocaryum urostachys (Palmae) in Amazonian Ecuador. Nordic Journal of Botany 22: 353-360.

Kahn, F. \& J.J. de. Granville. 1992. Palms in Forest Ecosystems of Amazonia. Ecological Studies vol. 95, Springer Verlag, Berlin, $215 \mathrm{p}$.

Kahn F. \& B. Millán. 1992. Astrocaryum (Palmae, Cocoeae, Bactridinae) in Amazonia. A preliminary treatment. Bulletin de 1'Institut Français d'Études Andines 21:459-531.

Kahn, F. and F. Moussa. 1994. Las palmeras del Perú: Colecciones, patrones de distribución geográfica, ecología, estatus de conservación, nombres vernáculos, utilizaciones. Instituto Francés de Estudios Andinos (IFEA), Lima. 180 pages.

Montufar R. \& J.C. Pintaud. 2006. Variation in species composition, abundance and microhabitat preferences among western Amazonian terra firme palm communities. Botanical Journal of the Linnean Society 151: 127-140.

Pintaud J.C. 2005. A journey along the Rio Napo. Palms 49: 8591.

Pintaud J.C., D. Gluchy \& B. Ludeña. 2003. Diversidad genética y filogenia molecular del género Astrocaryum (Arecaceae). Revista de la Pontificia Universidad Católica del Ecuador 71: 249-259.

Pintaud J.C. \& F. Kahn. 2002. Endémisme et spéciation radiative chez les palmiers de forêt dense humide: les Iguanurinae de Nouvelle-Calédonie et le genre Astrocaryum en Amazonie. Biosystema 20: 81-87.

Räsänen M., Salo J. \& R.Kalliola. 1987. Fluvial perturbance in the western Amazonian river basin: regulation by long-term sub-Andean tectonics. Science 238: 1398-1401.

Vallejo G., C. Vegas \& J.C. Pintaud. 2004. Estudio comparativo de la distribución de Astrocaryum macrocalyx Burret y Astrocaryum urostachys Burret en función de la topografía, drenaje y arquitectura del bosque en la Amazonía occidental (Ecuador y Perú). Lyonia 7(1): 99-106. 


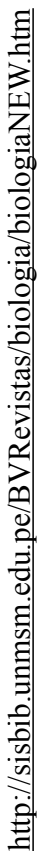

